\title{
Open
}

\section{Specific prebiotics modulate gut microbiota and immune activation in HAART-naive HIV-infected adults: results of the "COPA" pilot randomized trial}

\author{
A Gori $^{1}$, G Rizzardini ${ }^{2}$, B van't Land ${ }^{3,4}$, KB Amor ${ }^{3}$, J van Schaik ${ }^{3}$, C Torti ${ }^{5}$, T Quirino ${ }^{6}$, C Tincati ${ }^{7}$, \\ A Bandera ${ }^{1}, \mathrm{~J} \mathrm{Knol}^{3}$, $\mathrm{K}$ Benlhassan-Chahour $^{8}$, D Trabattoni ${ }^{9}$, D Bray ${ }^{8}$, A Vriesema ${ }^{3}$, G Welling ${ }^{10}$, \\ $\mathrm{J} \mathrm{Garssen}^{3,4}$ and M Clerici ${ }^{11}$
}

Intestinal mucosal immune system is an early target for human immunodeficiency virus type 1 (HIV-1) infection, resulting in $\mathrm{CD}^{+}{ }^{+} \mathrm{T}$-cell depletion, deterioration of gut lining, and fecal microbiota composition. We evaluated the effects of a prebiotic oligosaccharide mixture in highly active antiretroviral therapy (HAART)-naive HIV-1-infected adults. In a pilot double-blind, randomized, placebo-controlled study, 57 HAART-naive HIV-1-infected patients received a unique oligosaccharide mixture (15 or $30 \mathrm{~g}$ short chain galactooligosaccharides/long chain fructooligosaccharides/pectin hydrolysate-derived acidic oligosaccharides (scGOS/lcFOS/pAOS) daily) or a placebo for 12 weeks. Microbiota composition improved significantly with increased bifidobacteria, decreased Clostridium coccoides/Eubacterium rectale cluster, and decreased pathogenic Clostridium lituseburense/Clostridium histolyticum group levels upon prebiotic supplementation. In addition, a reduction of soluble CD14 (sCD14), activated CD4 $/$ CD25 + T cells, and significantly increased natural killer (NK) cell activity when compared with control group were seen in the treatment group. The results of this pilot trial highly significantly show that dietary supplementation with a prebiotic oligosaccharide mixture results in improvement of the gut microbiota composition, reduction of SCD14, CD4 ${ }^{+} \mathrm{T}$-cell activation (CD25), and improved NK cell activity in HAART-naive HIV-infected individuals.

\section{INTRODUCTION}

Human immunodeficiency virus (HIV) infection is characterized by progressive $\mathrm{CD} 4^{+} \mathrm{T}$-cell depletion and immunodeficiency that paradoxically occur in the context of a chronic state of immune system activation. Early in HIV infection, a profound $\mathrm{CD} 4{ }^{+} \mathrm{T}$-cell depletion is found in the intestinal mucosa, resulting in deterioration of gut homeostasis. ${ }^{1-3}$ The importance of gut-associated lymphoid tissue in the pathogenesis of HIV-1 infection has regained interest since Brenchley et al. ${ }^{4}$ found that gut-derived bacterial components in the blood of infected subjects are increased upon disease progression. Both gut immune activation and inflammation, as well as decreased mucosal repair and regeneration, contribute to
HIV-1-associated enteropathy, suggested to lead to increased bacterial compounds into the circulation. ${ }^{5}$ These bacterial components (lipopolysaccharide (LPS), peptidoglycan, and bacterial DNA) may further stimulate the vicious circle of immune activation, which in turn promotes viral replication and disease progression. After antiretroviral therapy initiation, activation of $\mathrm{CD} 4^{+} \mathrm{T}$ cells still occurs and is related to less increase of $\mathrm{CD} 4^{+} \mathrm{T}$ cells over time and predicts more rapid disease progression. ${ }^{6-8}$

Recently, we showed that impairment of the gastrointestinal (GI) tract in HIV-1-infected adults is already present in the early phases of HIV-1 disease. The presence of opportunistic pathogens like Pseudomonas aeruginosa and Candida albicans

\footnotetext{
${ }^{1}$ Division of Infectious Diseases, Department of Internal Medicine, "San Gerardo" Hospital, University of Milano-Bicocca, Monza, Italy. ${ }^{2}$ Department of Infectious Diseases and Tropical Medicine, "Luigi Sacco" Hospital, Milan Italy. ${ }^{3}$ Danone Research-Centre for Specialised Nutrition, Wageningen, The Netherlands. ${ }^{4}$ Division of Pharmacology, University of Utrecht, Utrecht, The Netherlands. ${ }^{5}$ Clinic of Infectious Diseases, University of Brescia, Brescia, Italy. ${ }^{6}$ Infectious Diseases Unit, Busto Arsizio Hospital, Busto Arsizio, Italy. ${ }^{7}$ Department of Internal Medicine, Clinic of Infectious Diseases, "San Paolo" Hospital, University of Milan, Milan, Italy. ${ }^{8}$ ImmunoClin, Paris, France. ${ }^{9}$ Chair of Immunology, DISP LITA Vialba, University of Milan, Milan, Italy. ${ }^{10}$ Department of Medical Microbiology, University Medical Center Groningen, Groningen, The Netherlands. ${ }^{11}$ Department of Biomedical Sciences and Technologies, University of Milan, and Laboratory of Molecular Medicine and Biotechnology, Don C. Gnocchi Foundation IRCCS, Milan, Italy. Correspondence: A Gori (andrea.gori@unimib.it) 
was found to be 10-fold and 10,000-fold increased, respectively, compared with levels reported in a healthy population. In addition, lower levels of beneficial microbial groups were found, such as bifidobacteria and lactobacilli, compared with levels reported for the general population. ${ }^{9}$ Both bifidobacteria and lactobacilli groups have a positive influence on mucosal immune function and gut health. ${ }^{10}$ In addition, McKenna et al. ${ }^{11}$ demonstrated altered gut microbiota composition in Simian immunodeficiency virus-infected macaques with colitis, strongly supporting the recent hypothesis that microbial alterations at GI-tract level are a key factor in the pathogenesis of chronic HIV infection. ${ }^{9,12,13}$ The gut microbiota represents a crucial line of resistance to colonization by pathogens, ${ }^{14}$ controls proliferation and differentiation of epithelial cells, ${ }^{15}$ and modulates maturation and activity of both innate and adaptive immune system. ${ }^{16,17}$ Impairment in microbiota composition can be addressed by using prebiotics. Prebiotics are nondigestible food ingredients, generally oligosaccharides, that modify intestinal microbiota balance by stimulating the growth of beneficial bacteria, such as bifidobacteria and lactobacilli. ${ }^{18}$ Prebiotic oligosaccharides can act indirectly through microbiota-dependent mechanisms (i.e., rebalancing microbiota composition in the gut) and/or have a direct effect via activation or blockage of cellular receptors. ${ }^{19}$ Prebiotic oligosaccharides can improve immune balance in infants, resulting in lower incidence of infections early in life, and correlate with improvement of intestinal microbiota with increased bifidobacteria levels. ${ }^{20,21}$ Given the imbalanced microbiota composition and altered immune function already present in the earlier stages of HIV-1 infection, we investigated in a pilot study the possible microbial- and immune-modulating effects of dietary supplementation with a unique mixture of prebiotic oligosaccharides in highly active antiretroviral therapy (HAART)-naive HIV-1-infected adults.

\section{RESULTS}

\section{Study population}

Between June 2005 and May 2006, 57 HAART-naive HIV-1positive adults were included and no differences between groups were observed with respect to baseline demographic and clinical characteristics (Table 1). Ten subjects did not complete the 12-week intervention period and were therefore excluded from per-protocol study group. Four subjects dropped out because of adverse events $\left(15 \mathrm{~g} \mathrm{day}^{-1}, n=3 ; 30 \mathrm{~g} \mathrm{day}^{-1}, n=1\right)$, two subjects withdrew consent $\left(15 \mathrm{~g} \mathrm{day}^{-1}, n=1 ; 30 \mathrm{~g} \mathrm{day}^{-1}, n=1\right)$, and four were lost to follow-up $\left(15 \mathrm{~g} \mathrm{day}^{-1}, n=1 ; 30 \mathrm{~g} \mathrm{day}^{-1}, n=1\right.$; control, $n=2)$.

\section{Product tolerability}

Both groups receiving the unique prebiotic mixture showed a nonsignificant increase in total GI score at week 4 of product intake compared with control group ( 1 and 2.5 , respectively, for 15 and $30 \mathrm{~g} \mathrm{day}^{-1}$ group). In the $30 \mathrm{~g} \mathrm{day}^{-1}$ group, complaints significantly increased at week 12 compared with baseline. The main complaints were flatulence and abdominal distension. At 4 weeks after last product intake, scores had returned again to 0 , similar to baseline (Table 2).

\section{Product safety}

Prebiotic supplementation did not result in any clinically relevant changes in biochemical safety parameters of liver function (plasma aspartate aminotransferase, alanine aminotransferase, $\gamma$-glutamyltransferase, and albumin) and renal function (plasma creatinine). Furthermore, no unexpected clinical adverse events (i.e., other than GI, product-related complaints, as discussed in the previous paragraph) or serious adverse events were observed in this HIV-1-infected treatment-naive study population.

Table 2 The composite GI symptom score (median (range)) for the ITT population $(n=57)$

\begin{tabular}{|c|c|c|c|}
\hline & $\begin{array}{c}\text { Controls } \\
(n=19)\end{array}$ & $\begin{array}{c}\text { scGOS/lcFOS/ } \\
\text { pAOS } 15 \text { g day }^{-1} \\
(n=19)\end{array}$ & $\begin{array}{c}\text { scGOS/lcFOS/ } \\
\text { pAOS } 30 \text { g day }^{-1} \\
(n=19)\end{array}$ \\
\hline Day $2 / 3$ & $0(0-2)$ & $0(0-3)$ & $0(0-8)$ \\
\hline Week 4 & $0(0-3)$ & $1(0-8)$ & $2.5(0-12)$ \\
\hline Week 12 & $0(0-6)$ & $1(0-6)$ & $2(0-6)^{a}$ \\
\hline Week 16 & $0(0-2)$ & $0(0-3)$ & $0(0-5)^{b}$ \\
\hline
\end{tabular}

Abbreviations: GI, gastrointestinal; ITT, intent-to-treat; IcFOS, Iong chain fructooligosaccharides; pAOS, pectin hydrolysate-derived acidic oligosaccharides; scGOS, short chain galactooligosaccharides.

asignificant difference $(P=0.010)$ between $30 \mathrm{~g} \mathrm{day}^{-1}$ dose and control group on within-subject change from week 12 to day $2 / 3$.

bSignificant difference $(P=0.019)$ between $30 \mathrm{~g} \mathrm{day}^{-1}$ dose and control group on within-subject change from week 16 to week 12 .

Table 1 Patient demographic and clinical characteristics (mean \pm s.d.) at baseline $(n=57)$

\begin{tabular}{|c|c|c|c|c|}
\hline & $\begin{array}{l}\text { Total group } \\
(n=57)\end{array}$ & $\begin{array}{c}\text { Control } \\
(n=19)\end{array}$ & $\begin{array}{c}\text { scGOS/lcFOS/pAOS } \\
15 \mathrm{~g} \mathrm{day}^{-1}(n=19)\end{array}$ & $\begin{array}{c}\text { scGOS/lcFOS/pAOS } \\
30 \text { g day }^{-1}(n=19)\end{array}$ \\
\hline $\operatorname{Sex}(M / F)$ & $42 / 15$ & $12 / 7$ & $13 / 6$ & $17 / 2$ \\
\hline Age (years) & $38.3 \pm 9.5$ & $39.3 \pm 12.1$ & $37.4 \pm 7.9$ & $38.2 \pm 8.2$ \\
\hline Height $(\mathrm{cm})$ & $172.0 \pm 8.2$ & $170.8 \pm 8.1$ & $172.0 \pm 9.1$ & $173.1 \pm 7.8$ \\
\hline Weight (kg) & $71.3 \pm 14.9$ & $68.3 \pm 16.9$ & $71.7 \pm 16.1$ & $73.8 \pm 11.4$ \\
\hline BMI $\left(\mathrm{kg} \mathrm{m}^{-2}\right)$ & $24.0 \pm 3.8$ & $23.4 \pm 4.8$ & $24.0 \pm 3.6$ & $24.6 \pm 3.2$ \\
\hline $\mathrm{CD}^{+}{ }^{+}$count (cells per $\left.\mu \mathrm{l}\right)$ & $520 \pm 161$ & $502 \pm 149$ & $536 \pm 173$ & $519 \pm 166$ \\
\hline HIV-1 RNA (copies per ml) & $28.4 \pm 40.5 \times 10^{3}$ & $28.9 \pm 33.6 \times 10^{3}$ & $22.21 \pm 46.3 \times 10^{3}$ & $34.8 \pm 41.2 \times 10^{3}$ \\
\hline
\end{tabular}

Abbreviations: BMI, body mass index; F, female; HIV-1, human immunodeficiency virus type 1; IcFOS, Iong chain fructooligosaccharides; M, male; pAOS, pectin hydrolysate-derived acidic oligosaccharides; scGOS, short chain galactooligosaccharides. 
One serious adverse event, syphilis, occurred that was not related to the product.

\section{Prebiotic effect of short chain galactooligosaccharides/long chain fructooligosaccharides/pectin hydrolysate-derived acidic oligosaccharides (scGOS/lcFOS/pAOS) on microbiota composition}

After 12 weeks, a significant increase in bifidobacteria population (median (range)) was found compared with baseline in both $15 \mathrm{~g} \mathrm{day}^{-1}$ (from $2.8 \%$ (0.0001-30.6) to $15.7 \%$ (1.2-36.4), $P=0.007$ ) and $30 \mathrm{~g} \mathrm{day}^{-1}$ groups (from $3.6 \%(0.000001-22.3)$ to $18.9 \%$ (0.9-46.9), $P=0.01$ ), but not in the control group (from $1.5 \%(0.0-24.9)$ to $5.2 \%(0.3-19.0))$ (Figure 1a). This change from baseline in both groups receiving prebiotic intervention was significantly higher $\left(P=0.009\right.$ for $15 \mathrm{~g} \mathrm{day}^{-1}$ group and $P=0.015$ for $30 \mathrm{~g} \mathrm{day}^{-1}$ group) compared with the control group. In addition, a significant higher level of bifidobacteria population was detected at week 12 for groups receiving $15 \mathrm{~g} \mathrm{day}^{-1}$ $(P=0.014)$ and $30 \mathrm{~g} \mathrm{day}^{-1}(P=0.007)$ dose, respectively, compared with the control group, suggesting improvement of bifidobacterial levels as depicted in Figure 1a.

In the higher-dose group of prebiotics $\left(30 \mathrm{~g} \mathrm{day}^{-1}\right)$, a significant decrease in C. lituseburense/C. histolyticum group, which includes pathogenic Clostridium perfringens and Clostridium difficile species (estimated marginal mean (95\% confidence interval)), was found compared with baseline levels (from $0.016 \%$ (0.004-0.064) to $0.002 \%$ (0.001-0.007), $P=0.009)$ (Figure 1b). In contrast, a significant increase (from $0.011 \%(0.003-0.041)$ to $0.044 \%(0.015-0.125), P=0.030)$ was observed in the control group. Moreover, at week 12, a significant lower level of C. lituseburense/C. histolyticum group was detected in both 15 and $30 \mathrm{~g} \mathrm{day}^{-1}$ dose groups $(P=0.011$ and $P<0.001$, respectively) compared with the control group. In addition, a significant decrease from baseline of Eubacterium rectale/Clostridium coccoides cluster was found in both $15 \mathrm{~g} \mathrm{day}^{-1}(P=0.035)$ and $30 \mathrm{~g} \mathrm{day}^{-1}$ dose groups $(P=0.05)$ (Figure 1c), whereas no change from baseline was seen in the control group. There were no changes found in other measured intestinal bacterial groups, including P. aeruginosa, C. albicans, Bacteroides/ Prevotella, lactobacilli, Escherichia coli, and Atopobium.

\section{Prebiotic effect of scGOS/lcFOS/pAOS on plasma LPS and soluble CD14 (sCD14) concentration}

The levels of sCD14 were measured in plasma of all patients at baseline as well as after 4,12 , and 16 weeks of prebiotic intervention. The analysis of sCD14 levels in plasma showed that patients treated with $15 \mathrm{~g} \mathrm{day}^{-1}$ had significantly lower levels after 4 and 12 weeks of treatment compared with placebo group (week 4: 9,952 $\mathrm{pg} \mathrm{m}^{-1}$ (s.d. $\pm 1,245$ ) vs. $11,237 \mathrm{pg} \mathrm{ml}^{-1}$ (s.d. $\pm 1,342$ ), $P=0.02$; week $12: 9,720 \mathrm{pg} \mathrm{ml}^{-1}$ (s.d. $\pm 1,442$ ) vs. $11,302 \mathrm{pg} \mathrm{ml}^{-1}$ (s.d. $\pm 1,721$ ), $P=0.02$ ) and significantly lower levels after 12 weeks compared with patients treated with $30 \mathrm{~g} \mathrm{day}^{-1}\left(11,244 \mathrm{pg} \mathrm{ml}^{-1}\right.$ (s.d. $\left.\left.\pm 1,997\right), P=0.04\right)$ (Figure 2).

LPS analysis did not show differences between treatment groups; however, patients treated with $15 \mathrm{~g} \mathrm{day}^{-1}$ showed a significant decrease in LPS levels at week 16 compared with
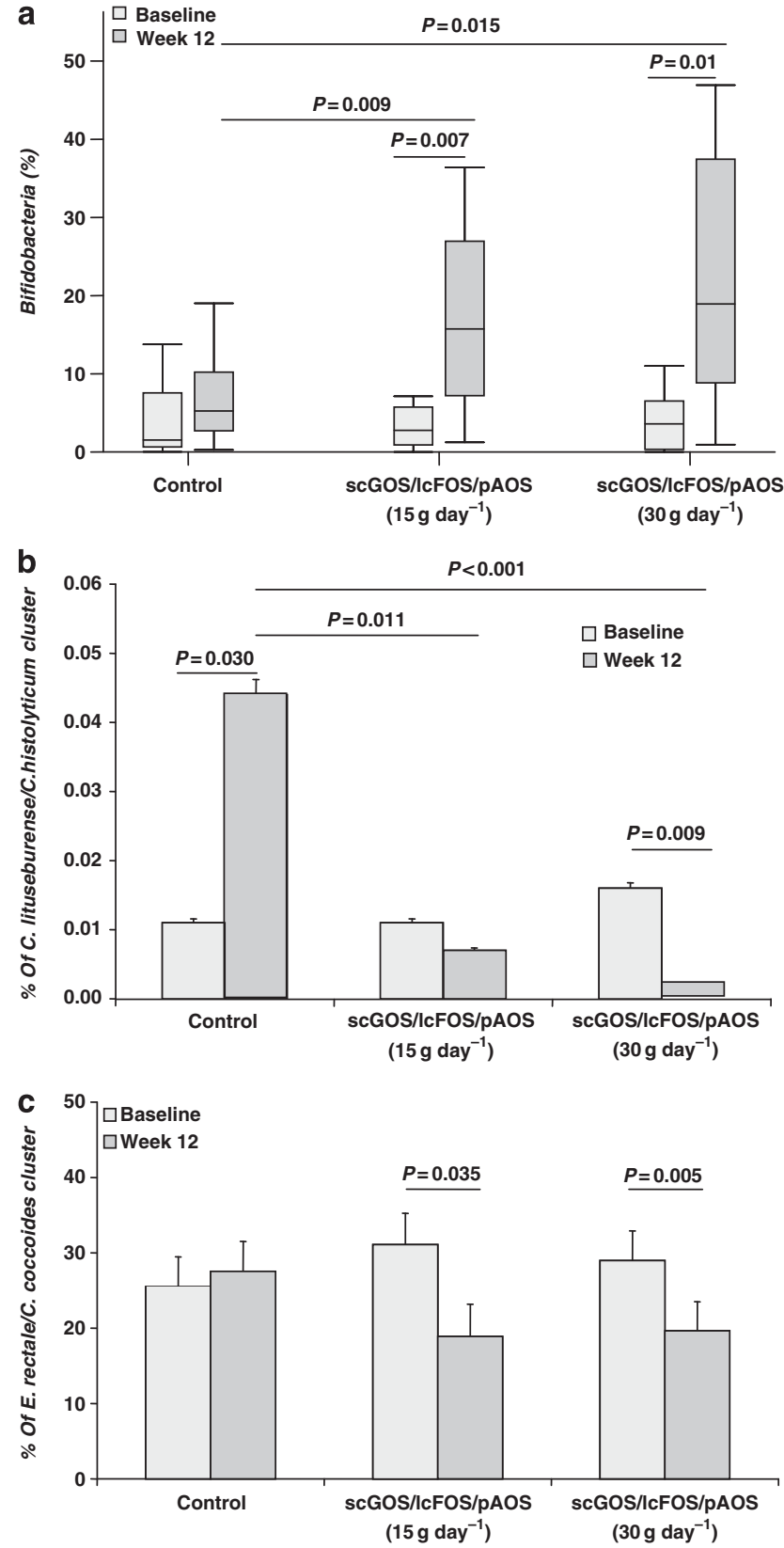

Figure 1 Improved gastrointestinal (GI) microbiota composition upon 12-week intake of short chain galactooligosaccharides/long chain fructooligosaccharides/pectin hydrolysate-derived acidic oligosaccharides (scGOS/lcFOS/pAOS). A 12-week prebiotic intervention with scGOS/lcFOS/pAOS in highly active antiretroviral therapy (HAART)-naive human immunodeficiency virus type 1 (HIV-1)infected individuals was associated with (a) increased bifidobacterial levels expressed as median (range) percentage of total fecal bacteria, (b) reduced levels of the pathogenic Clostridium histolyticum cluster expressed as estimated marginal mean percentage $( \pm$ s.e.) of the total fecal bacteria, and (c) reduced levels in the Eubacterium rectale/Clostridium coccoides cluster expressed as estimated marginal mean percentage $( \pm$ s.e.) of total fecal bacteria. $P$-values indicate significant changes from either baseline within the groups as well as between groups (as tested with Mann-Whitney, analysis of variance (ANOVA)). 


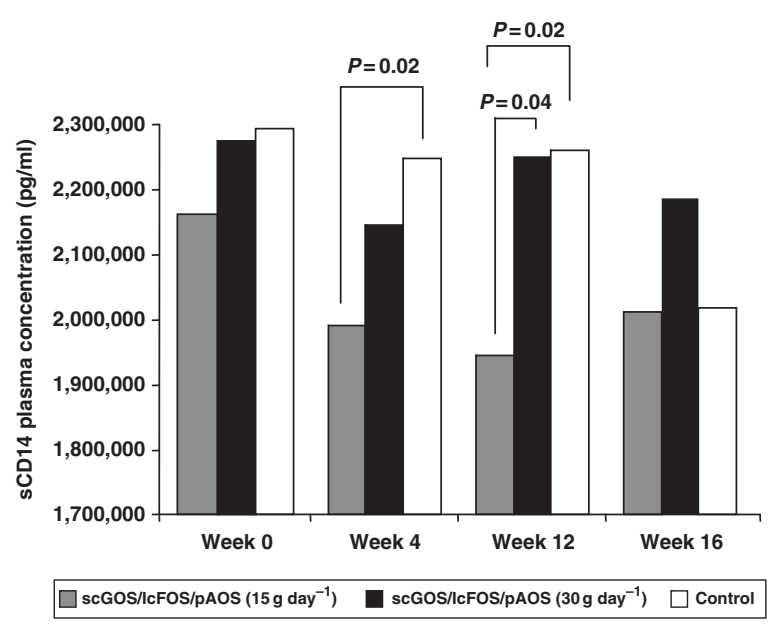

Figure 2 Effective reduction on plasma soluble CD14 (sCD14) concentration after administration of short chain galactooligosaccharides/ long chain fructooligosaccharides/pectin hydrolysate-derived acidic oligosaccharides (scGOS/lcFOS/pAOS). Evaluation of SCD14 plasma levels at baseline and after 4,12 , and 16 weeks of prebiotic intervention. The analysis of SCD14 levels in plasma showed that patients treated with $15 \mathrm{~g} \mathrm{day}^{-1}$ had significantly lower levels after 4 and 12 weeks of treatment compared with placebo group and significantly lower levels after 12 weeks compared with patients treated with $30 \mathrm{~g} \mathrm{day}^{-1}$.

baseline values $\left(519.5 \mathrm{pg} \mathrm{ml}^{-1}\right.$ (s.d. \pm 59.2$)$ vs. $588.2 \mathrm{pg} \mathrm{ml}^{-1}$ (s.d. \pm 104.5$), P=0.04)$.

\section{scGOS/IcFOS/pAOS reduces immune activation of CD4 ${ }^{+} T$ cells}

Although HIV-1 plasma viral load changes were found in groups receiving prebiotics (log viral load changed from $3.91 \pm 0.21$ to $3.73 \pm 0.20\left(15 \mathrm{~g} \mathrm{day}^{-1}\right)$ and from $4.28 \pm 0.24$ to $3.98 \pm 0.21$ $\left(30 \mathrm{~g} \mathrm{day}^{-1}\right)$ (estimated marginal mean $( \pm$ s.e.m. $)$ ) when compared with control (log viral load changed from $4.06 \pm 0.22$ to $4.07 \pm 0.19$ estimated marginal mean $( \pm$ s.e.m. $)$ ), these were not statistically significant (Table 3 ). CD $4^{+} \mathrm{T}$-cell count did not change upon the short 12 weeks of dietary prebiotic supplementation, neither between groups nor within groups over time, as shown in detail in Table 3. Nevertheless, a dose-dependent reduction of activated $\mathrm{CD} 4{ }^{+} / \mathrm{CD} 25^{+} \mathrm{T}$ cells, expressed as percentage of total lymphocytes, was detected upon 12 weeks of prebiotic intervention (Figure 3). Subjects receiving the lower dose $\left(15 \mathrm{~g} \mathrm{day}^{-1}\right)$ showed a clear trend toward reduced levels of activated $\mathrm{CD} 4{ }^{+} / \mathrm{CD} 25^{+} \mathrm{T}$ cells (median (min-max)) after 12 weeks compared with baseline levels (from 0.36\% (0.04-1.9) to $0.22 \%(0.02-1.25), P=0.09)$, whereas the higher-dose group $\left(30 \mathrm{~g} \mathrm{day}^{-1}\right)$ showed a statistically significant reduction compared with baseline (from $0.52 \%(0.01-7.21)$ to $0.27 \%(0.02-1.54)$, $P<0.01)$. Compared with the control group, the group receiving $15 \mathrm{~g} \mathrm{day}^{-1}$ dose showed statistically significant lower CD4 ${ }^{+}$ T-cell activation $(\mathrm{CD} 25)$ at week $12(P<0.05)$. The reduction in activated $\mathrm{CD} 4{ }^{+} / \mathrm{CD} 25^{+} \mathrm{T}$-cell levels was not accompanied by a change from baseline in levels of activated $\mathrm{CD} 8^{+} \mathrm{T}$ cells (as measured by percentage of $\mathrm{CD} 8{ }^{+} / \mathrm{CD} 38^{+} / \mathrm{CD} 45 \mathrm{RO}^{+} \mathrm{T}$ cells) nor by change from baseline in levels of FoxP3 (forkhead box
P3)-expressing regulatory $\mathrm{CD} 4^{+} / \mathrm{CD} 25^{++} \mathrm{T}$ cells. Also, no significant changes from baseline were found in $\mathrm{B} 7-\mathrm{H} 1$ percentages on either monocytes or B cells (Table 3) or in cytokine profiles upon ex vivo re-stimulation with gag peptides or phytohemagglutinin (data not shown).

\section{scGOS/IcFOS/pAOS improves natural killer (NK) cell activity}

In addition to significant changes in the levels of activated $\mathrm{CD} 4^{+} / \mathrm{CD} 25^{+} \mathrm{T}$ cells, NK cell activity significantly improved upon prebiotic intervention. The strongest effect was observed in the group receiving $15 \mathrm{~g} \mathrm{day}^{-1}$ dose, with significant increases in all effector-target (E/T) ratios tested (12.5:1, 25:1, and 50:1) with respectively 4.25 -fold $(P=0.002) 3.63$-fold $(P=0.001)$, and 3.44-fold $(P<0.001)$ higher levels compared with baseline (Figure 3). Similar effects, although not statistically significant for two of three ratios tested, were observed in the group receiving $30 \mathrm{~g} \mathrm{day}^{-1}$ dose, in whom NK activity was increased from baseline 2.15-fold ( $P=0.026), 2.01$-fold $(P=0.083)$, and 1.93-fold $(P=0.107)$ for $\mathrm{E} / \mathrm{T}$ ratios $12.5: 1,25: 1$, and 50:1, respectively. No differences were found in control group compared with baseline. In addition, in the group receiving $15 \mathrm{~g} \mathrm{day}^{-1}$ dose, NK cell activity at week 12 was significantly improved compared with subjects receiving control product at $\mathrm{E} / \mathrm{T}$ ratios of 25:1 $(P=0.006)$ and 50:1 $(P=0.003)$.

\section{DISCUSSION}

Data herein suggest that dietary supplementation with a unique mixture of prebiotic oligosaccharides, consisting of scGOS/ lcFOS/pAOS, may positively modulate gut microbiota composition, resulting in decreased sCD14 and LPS levels, CD4 ${ }^{+}$ T-cell activation (CD25), and increased activity of NK cells in HAART-naive HIV-infected adults.

The epithelial surface of the intestine is colonized by a high number of bacteria communities considered to be the first component of the defensive gut barrier. It represents a crucial line of resistance to colonization by pathogens, thereby decreasing the likelihood of bacterial translocation. Improved growth of bifidobacteria upon prebiotic feeding leads to a decrease in the $\mathrm{pH}$ and modulation of the short-chain fatty acids pattern that could contribute to the protection against pathogens. This study shows that supplementation with scGOS/lcFOS/pAOS mixture increased significantly the level of bifidobacteria in both intervention groups and reduced the levels of pathogenic clostridia-related species. Despite the reduced levels of pathogenic clostridia-related species, a full reduction of pathogenic load upon 12 weeks of intervention was not achieved, as levels of other pathogenic species like $P$. aeruginosa or $C$. albicans remained unchanged. In both prebiotic groups, a reduction in the level of E. rectale/ C. coccoides cluster was observed. This bacterial cluster represents almost $75 \%$ of the Firmicutes, a major group of gut microbiota, including most of butyrate producers and has recently been implicated to play a role in human energy sequestration from diet. ${ }^{16}$ Although specific relevance of alterations within this cluster to HIV-1 infection remains unclear, alterations found in this study are indicative for rebalancing intestinal microbiota 


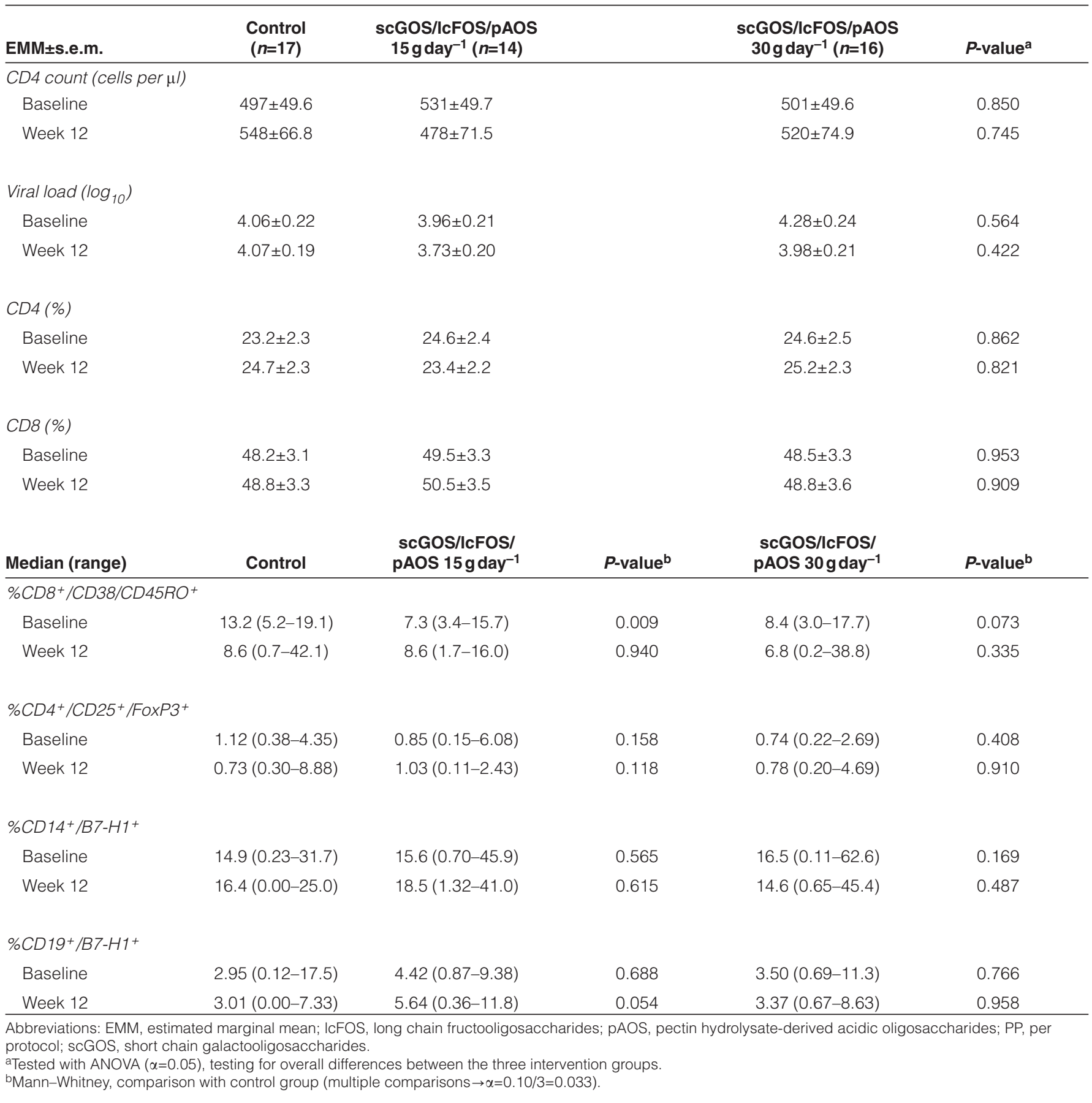

in treatment-naive HIV-1 infected upon supplementation with the specific prebiotic mixture. This reassess is also confirmed by the significant decrease of sCD14 plasma levels in the patients receiving $15 \mathrm{~g} \mathrm{day}^{-1}$ compared with placebo group and by the reduction of LPS plasma levels at week 16 in patients treated with $15 \mathrm{~g} \mathrm{day}^{-1}$ compared with baseline values.

Although in this study a healthy control group was not included, we recently reported Bifidobacterium levels to be lower in HAART-naive HIV-1-infected adults than those reported in the general population. ${ }^{9}$ Modulating the GI tract toward a bifidogenic microbiota has long been regarded as a beneficial health effect for the host. Bifidobacteria supplementation has been associated with lower bacterial translocation, leading to a decrease in the inflammatory cascade activation in several models of bacterial translocation. ${ }^{22}$ Similarly, other studies have demonstrated that modulation of the gut microbiota via prebiotic or probiotic ingestion may improve or prevent disruption of intestinal permeability in animal models and humans. ${ }^{23}$ Furthermore, increased levels of fecal bifidobacteria have been shown to reduce intestinal LPS in murine 

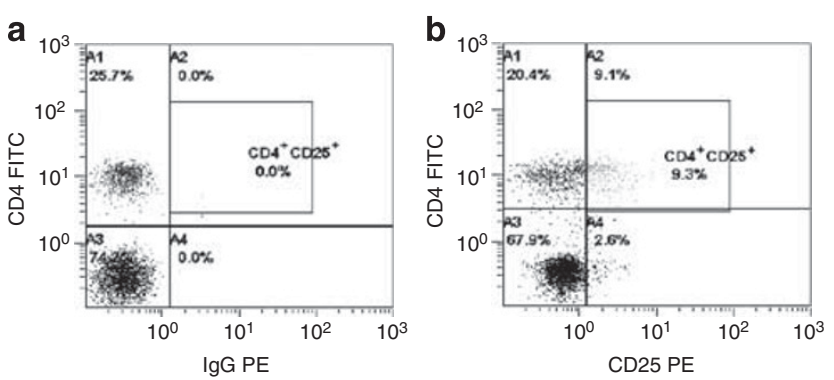

。

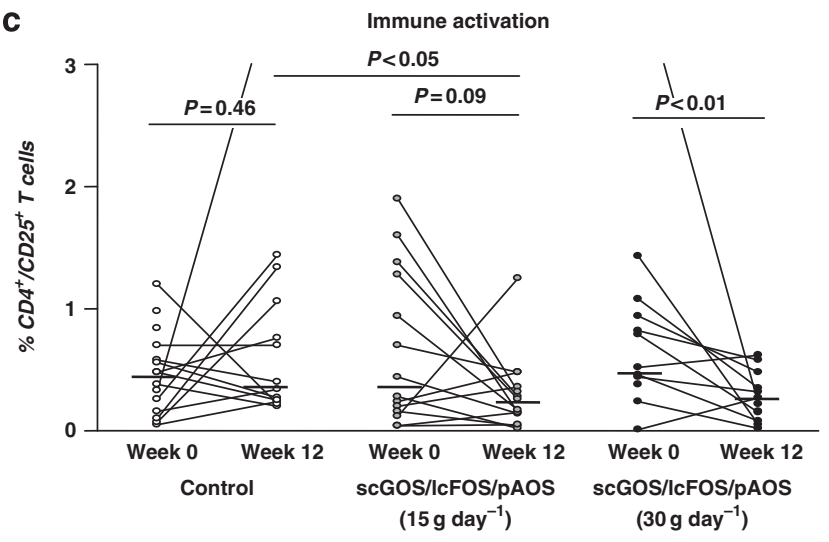

d

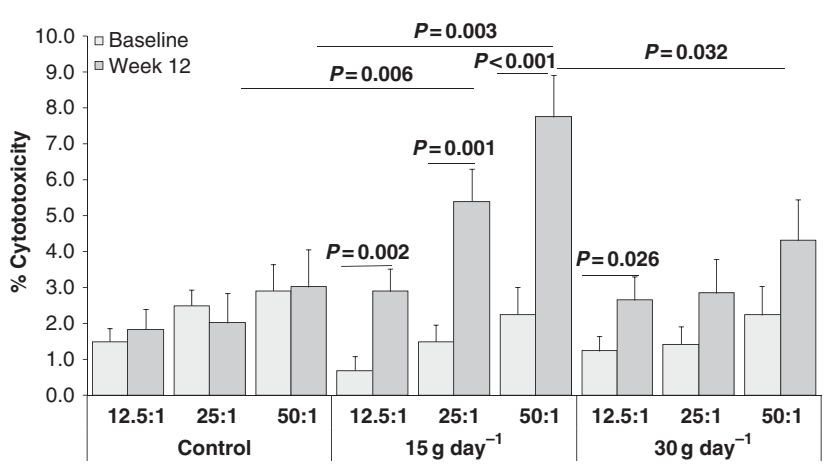

Figure 3 Beneficial immune changes because of intake of short chain galactooligosaccharides/long chain fructooligosaccharides/pectin hydrolysate-derived acidic oligosaccharides (scGOS/lcFOS/pAOS). Flow cytometry measurement of CD25 expression on CD4 ${ }^{+} \mathrm{T}$ cells on lymphocytes first gated on CD45 and CD3. In (a) cells are visualized labeled with isotype control, whereas (b) represents the CD25 labeling. The 12-week scGOS/LcFOS/pAOS intake was associated with (c) reduced $\mathrm{CD} 4{ }^{+} \mathrm{T}$-cell activation and $(\mathbf{d})$ improved natural killer (NK) cell cytotoxicity. Individual percentages of $\mathrm{CD} 4^{+} / \mathrm{CD} 25^{+} \mathrm{T}$ cells are shown as dots, with median values displayed per group as lines. The $P$-values indicate statistical significant changes from baseline as tested with Mann-Whitney ( $\alpha=0.033$ for three multiple comparisons). Percentage cytotoxicity is expressed as estimated marginal means ( \pm s.e.) of target cell lysis at indicated effector-target $(\mathrm{E} / \mathrm{T})$ ratios. The $P$-values indicate significant changes from baseline within the groups as well as between groups (analysis of variance (ANOVA)).

models and to improve the mucosal barrier function. ${ }^{24}$ Thus, particular bacterial species from the intestinal microbiota, including bifidobacteria, may well possess immunomodulatory properties during HIV-1 infection, although that study population was different compared with the HAART-naive subjects in
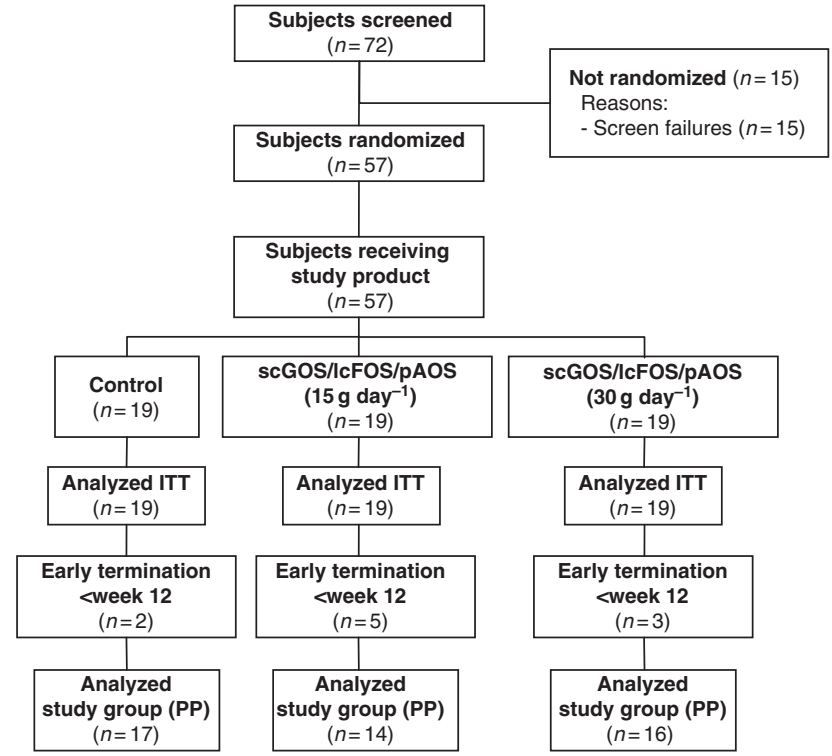

Figure 4 Overview of patient inclusion. Patients were randomized based on study in and exclusion criteria and evenly divided over the three arms. Patients who did not complete the 12-week supplementation period were excluded from the per-protocol (PP) study group and analyzed in the intent-to-treat (ITT) study group. IcFOS, long chain fructooligosaccharides; pAOS, pectin hydrolysate-derived acidic oligosaccharides; scGOS, short chain galactooligosaccharides.

our study. ${ }^{25-28}$ In addition, probiotic supplementation has been indicated to possibly influence CD4 count. ${ }^{29}$

As it is well recognized that translocation of luminal bacteria and toxins is linked namely to disruption of the normal balance in the gut microbiota, impaired immune function and gut barrier function, ${ }^{30}$ it is postulated that the clinical benefits from consumption of prebiotics are obtained through their effect on the colonic microbiota or directly through the immune system. In addition to the observed intestinal microbiota changes in our study, a clear dose-dependent inhibition of $\mathrm{CD} 4{ }^{+} \mathrm{T}$-cell activation, although only measured by CD 25 expression, was demonstrated. Although no statistically significant effect of the intervention was found on $\mathrm{CD} 4^{+} \mathrm{T}$-cell levels in HIV-1-infected adults and only a slight reduction $(0.2-0.3 \mathrm{log})$ in viral load was observed. T-cell activation in our study was measured by the expression of CD38, memory $\mathrm{CD} 45 \mathrm{RO}^{+} \mathrm{CD}^{+}{ }^{+} \mathrm{T}$ cells, and $\mathrm{CD} 25$ on $\mathrm{CD} 8{ }^{+}$and $\mathrm{CD} 4{ }^{+}$ $\mathrm{T}$ cells, respectively. In contrast to the reduced $\mathrm{CD} 4^{+} \mathrm{T}$-cell activation, the $\mathrm{CD} 38$ expression on $\mathrm{CD}^{+}{ }^{+} \mathrm{T}$ cells was not altered, although these data may have been distorted because of high variation and differences in activation of CD38 by memory $\left(\mathrm{CD} 4 \mathrm{RO}^{+}\right) \mathrm{CD}^{+} \mathrm{T}$ cells, a measure of pathologic immune activation in HIV-1 infection associated with disease progression (Table 3 ).

In light of the critical role of regulatory $\mathrm{T}$ cells in the regulation of immune cells, and suggested role in immunopathology during chronic HIV-1 infection also, the number of regulatory T cells was measured in our study. A rapid disease progression seems to be associated with the expansion of regulatory $\mathrm{T}$ cells 
in the course of HIV-1 infection. In addition, Cao et al. ${ }^{31}$ recently suggested that $\mathrm{CD} 4^{+}$, but not $\mathrm{CD} 8^{+}$, T-cell activation was correlated with the proportion of regulatory $\mathrm{T}$ cells in HIV-1-infected individuals. ${ }^{31}$ The present results did not indicate any modification in the number of $\mathrm{CD} 4^{+} / \mathrm{CD} 25^{+} /$ Foxp $3^{+}$regulatory $\mathrm{T}$ cells as result of 12 weeks of prebiotic intake.

One of the notable findings from our preliminary study is that upon prebiotic supplementation, a significant improvement in NK cell cytotoxicity was found, with the most pronounced effect in $15 \mathrm{~g} \mathrm{day}^{-1}$ dose group. This suggests that for NK cell improvement the effect can already be reached using $15 \mathrm{~g} \mathrm{day}^{-1}$ and there is no additive value on this marker to use the $30 \mathrm{~g} \mathrm{day}^{-1}$ dose. In HIV-1 infection in humans, an inverse association exists between viral load/replication during chronic infection and both NK cell frequency and function. ${ }^{32-35}$ Our finding might be explained by reduced levels of translocated bacterial components from the gut upon re-establishment of the gut microbial ecosystem, as chronic exposure to LPS, which is known to be related to reduced NK cell cytotoxicity. ${ }^{36}$ Furthermore, programmed cell death-1 (PD-1)/PD-1L pathway, was previously found to be associated with NK and T-cell dysfunction, in that upregulation of PD-1 expression in rapid progressors is associated with a reduced cytolytic activity, i.e., perforin and interferon- $\gamma$ production. ${ }^{37}$ However, no significant changes were observed on either B7-H1 expression on monocytes nor on $\mathrm{CD}^{+} \mathrm{T}$-cell activation upon 12 weeks of product intake. Taken together, the mechanism explaining the significant improvement in NK cell cytotoxicity as well as the reduction of HIV-1-induced immune activation by prebiotics remains to be elucidated.

To confirm acceptance of prebiotic supplementation in HIV-1-infected adults, tolerability and safety of scGOS/lcFOS/ pAOS mixture upon prolonged intake (12 weeks) was determined. In general, prebiotics might lead to gut symptoms including flatulence and abdominal distension. Therefore, initial increase observed in total GI score at week 4 was anticipated. The increase however was nonsignificant, and in general only "mild" scores in GI symptoms were recorded from subjects. Furthermore, a decrease in GI symptom incidence at the end of intervention was found compared with the incidence at week 4, which may well be explained by adaptation of microbiota composition and therefore improved/adapted fermentation of prebiotics. Apart from the observed GI symptoms, no other safety issues were identified (as demonstrated by liver and renal safety markers) with supplementation of this unique prebiotic mixture in HAART-naive HIV-1-infected adults. Therefore, the tested prebiotic mix can be regarded as tolerable and safe for this particular target population.

In conclusion, our study, although preliminary, suggests that in nonsymptomatic HAART-naive HIV-1-infected adults, gut microbiota can at least partially be restored by a unique prebiotic mixture consisting of scGOS/lcFOS/pAOS, with stimulation of bifidobacteria growth and reduction in fecal pathogenic load. In addition, we demonstrated a significant reduction in SCD14 and LPS levels, $\mathrm{CD} 4{ }^{+} \mathrm{T}$-cell activation (CD25), and improved NK cell cytolytic activity in these HIV-1-infected adults after prebiotic supplementation. The mechanisms responsible for these observations remain to be elucidated as well as persistence of these effects upon prolonged supplementation. Although prebiotic dietary fibers have been extensively investigated for their therapeutic effects in other settings, to our knowledge this is the first study demonstrating clear health beneficial effects of prebiotics in HIV-1 infection. Despite that statistically significant differences were observed, the data presented herein came from a small sample size patient cohort. It will therefore be important to validate these results in a larger cohort of HIVinfected individuals.

\section{METHODS}

\section{Study design}

In a double-blind, randomized, placebo-controlled, pilot study (COPA trial: Clinical trial with Oligosaccharides Powder for Application in HIV-1 infection), 57 nonsymptomatic HAART-naive HIV-1-positive adults were recruited in three Italian centers. Subjects were randomized in three groups, receiving three sachets of powder ( $16 \mathrm{~g}$ per sachet) daily. In two of these groups, the powder contained three different prebiotic oligosaccharide materials, i.e., scGOS (Borculo Domo, Zwolle, The Netherlands; 45\% scGOS), lcFOS (Orafti, Wijchen, The Netherlands; $100 \%$ lcFOS), and pAOS (Sudzucker, Mannheim, Germany; $85 \%$ galacturonic acid). The prebiotics were mixed in a ratio of 9:1:10 based on prebiotic purity, with one group receiving $15 \mathrm{~g} \mathrm{day}^{-1}$ and the other $30 \mathrm{~g} \mathrm{day}^{-1}$ of these prebiotics. The control group received solely digestible sugar maltodextrin. In order to get accustomed to the oligosaccharides, which could lead to GI effects like flatulence, subjects gradually increased product intake with the complete daily dose from the third week onward. Total study duration was 16 weeks, consisting of 12 weeks of intervention and 4 weeks of follow-up. Product tolerability and safety were assessed on the intent-to-treat study group. In order to analyze both immune and microbiota responses related to the intervention, these parameters were analyzed from those subjects who completed the 12-week supplementation period as depicted in more detail in Figure 4. All analysis and laboratory staff members were blinded during the entire duration of the study. The study was approved by the institutional review board of the "Luigi Sacco" Hospital, Milano, Italy, by the institutional review board of the Busto Arsizio Hospital, Busto Arsizio, Italy, and by the institutional review board of the Spedali Civili di Brescia Hospital, Brescia, Italy. The study has been conducted in compliance with "Declaration of Helsinki" principles and with local Italian laws and regulations. Written informed consent was obtained from all subjects before study participation.

\section{Product tolerability and safety}

GI tolerability was assessed on days 2 or 3 from baseline and at weeks 4, 12 , and 16 by a 4 -point scale recall questionnaire based on Van Aerde et al. ${ }^{38}$ Seven GI symptoms (nausea, burping, abdominal distention, flatulence, diarrhea, constipation, and urgent need for defecation) were each scored by the patients on " 0 " (no complaint) to " 3 " (severe complaint) scale. Total GI score was calculated as the sum of all scores. For product safety, liver function (plasma aspartate aminotransferase, alanine aminotransferase, $\gamma$-glutamyltransferase, and albumin) and renal function (plasma creatinine) were assessed at baseline and week 12 .

\section{Gut microbiota composition}

Fecal sample collection and preparation. Stool samples, collected at baseline and after 12 weeks, were immediately stored at $-20^{\circ} \mathrm{C}$ until further processing. Frozen samples were thawed on ice water and $0.5 \mathrm{~g}$ was fixed with paraformaldehyde as described previously. ${ }^{39}$ For DNA extraction, $0.2 \mathrm{~g}$ fecal sample was resuspended in $0.05 \mathrm{M}$ phosphate-buffered saline. DNA was isolated using bead beating method as described previously. ${ }^{39}$ 
Real-time PCR. Quantification of total bifidobacteria, lactobacilli, and $P$. aeruginosa was performed with real-time PCR using duplex $5^{\prime}$-nuclease assay. Measurement of bifidobacteria and lactobacilli was performed as previously described. ${ }^{39,40} P$. aeruginosa was determined using the method described by Pirnay et al. ${ }^{41}$ with some minor modifications; fluorescent labels were changed to 6FAM and TAMRA. Fluorescence signal was measured in annealing phase on ABI 7900HT Fast (Applied Biosystems, Nieuwerkerk a/d IJssel, The Netherlands). Total bacterial load was determined as described by Nadkarni et al. ${ }^{42}$ Relative percentage of $P$. aeruginosa was subsequently calculated using comparative Ct method according to Liu et al. ${ }^{43}$

Fluorescent in situ hybridization. Oligonucleotide probes used in this study were: (i) Chis 150 to detect C. histolyticum group, ${ }^{44}$ (ii) Clit135 for C. lituseburense group, ${ }^{44}$ (iii) Bac303 for Bacteroides/Prevotella group, ${ }^{45}$ (iv) Erec 482 for members of E. rectale/C. coccoides cluster, ${ }^{44}$ (v) Eco1531 for E. coli and related species, ${ }^{46}$ (vi) Caal for C. albicans, ${ }^{47}$ and (vii) Ato291 for Atopobium cluster. ${ }^{48}$ These probes were covalently linked at their $5^{\prime}$-end either to fluorescein isothiocyanate (FITC) or Cy3. Fixed fecal samples were hybridized with probes as described previously. ${ }^{49}$ Fluorescent cells were then counted automatically ${ }^{50}$ with a Leica DMRXA epifluorescence microscope (Leica, Wetzlar, Germany). For each analysis, 25 microscopic fields were counted and the target bacterial groups were recorded as a percentage of total bacterial cell count. ${ }^{51}$

\section{Immunological markers}

Blood collection, $\mathrm{CD4}^{+} \mathrm{T}$-cell counts, and viral load measurements. Whole blood was collected by venipucture in Vacutainer tubes containing EDTA (BD Biosciences, Erembodegem, Belgium). CD4 ${ }^{+}$T-cell counts were determined by flow cytometry. Plasma HIV-1 RNA levels were quantified by nucleic acid signal-amplification assay with detection limit of 50 copies per ml. Peripheral blood mononuclear cells (PBMCs) were separated on lymphocyte separation medium (Organon Teknika, Dublin, Ireland), and washed twice in phosphate-buffered saline. Freshly isolated PBMCs were kept at room temperature and used within $12 \mathrm{~h}$. Additionally, PBMCs were also frozen in dimethyl sulfoxide and stored in liquid nitrogen until further use (see also FoxP3 staining and NK cell activity).

Immunophenotypic analyses. Lymphocyte subsets were evaluated by flow cytometric analysis, using $50 \mu$ lof EDTA peripheral blood incubated for $30 \mathrm{~min}$ at $4^{\circ} \mathrm{C}$ with fluorochrome-labeled monoclonal antibodies (CD4 R-PE-Cyanine 5 Tandem-PE-Cy5-; CD3 PE-Cy5; CD8 PE-Cy5; CD14 PE-Cy5; CD19 PE-Cy5; CD25 PE; CD38 PE; CD8 FITC; CD45RO FITC) (Caltag Laboratories, Burlingame, CA). After incubation, erythrocyte lysis and fixation was performed using Immuno-Prep EPICS kit (Coulter Electronics, Milano, Italy) and Q-prep Work Station (Coulter Electronics). For indirect immunofluorescence staining, freshly isolated PBMCs were incubated with mouse-anti-human B7-H1 antibody $\left(2.5 \mu \mathrm{g} \mathrm{m}^{-1}\right)$ a gift of Dr L. Chen (Johns Hopkins University School of Medicine, Baltimore, MD). After $30 \mathrm{~min}$ at $4^{\circ} \mathrm{C}$, cells were washed and incubated for $30 \mathrm{~min}$ at $4^{\circ} \mathrm{C}$ with rat-anti-mouse IgG $(\mathrm{H}+\mathrm{L}) \mathrm{Ab}$-FITC $\left(1 \mu \mathrm{g} \mathrm{ml}^{-1}\right)$ or with a mouse-anti-hamster IgG-PE $\left(1 \mu \mathrm{g} \mathrm{ml}^{-1}\right)($ Caltag Laboratories).

Cytometric analysis. Cytometric analyses were performed using an EPICS XL flow cytometer (Beckman Coulter, Brea, CA). For each analysis, 20,000 events were acquired and gated on CD4 (or CD8, CD14, CD19) expression and side scatter properties. For FoxP3 intracellular staining, cryopreserved PBMCs were thawed and washed with phosphatebuffered saline containing $2 \%$ bovine serum albumin. Subsequently, $1 \times 10^{6}$ cells were stained according to the manufacturer's protocol (eBioscience, San Diego, CA). Acquisition of data was performed on the same day as staining.
Plasma LPS and sCD14 concentration. LPS and sCD14 concentration was measured on plasma samples using LAL Chromogenic Endopoint Assay (Hycult Biotechnology, Uden, The Netherlands) and sCD14 ELISA kit (R\&D Systems, Minneapolis, MN; Bender MedSystems, Vienna, Austria), respectively. All the analyses were conducted following the manufacturer's instructions. Plasma concentration of each protein was calculated relatively to standard curve.

NK cell cytotoxicity. Cytotoxic activity of NK cells against target cell line (K562) was measured by flow cytometry using NKTESTkit in accordance with the manufacturer's instructions (ORPEGEN Pharma, Heidelberg, Germany). In brief, K562-target cells were labeled with lipophilic green fluorescent membrane dye discriminating effector and target cells. Cryopreserved PBMCs were thawed, washed, and subsequently incubated at ET ratios of 50:1, 25:1, and 12.5:1. After $4 \mathrm{~h}$ of incubation, killed target cells are identified by DNA stain. Percentage of target cells killed by effector NK was determined using FC 500 (Beckman Coulter).

\section{Statistical analyses}

Comparisons are made between results obtained at week 12 compared with baseline for all three groups. Also, change from baseline was compared between control group and the two treatment groups. The statistical analyses used for these comparisons are analysis of variance and Dunnett's test for multiple comparisons using SPSS (SPSS, Chicago, IL) version 12.1 or higher. For two comparisons, $P$-values of $<0.05$ were considered to be statistically significant, and for three comparisons, $\alpha$ of 0.10 was used. In case data were not normally distributed, log transformations were performed to obtain normal distributions and values are reported as estimated marginal mean with s.e.m. When transforming the data did not result in normal distribution of the parameter, nonparametrical testing (Mann-Whitney) was performed and values are reported as median with range.

\section{DISCLOSURE}

Belinda van't Land, Kaouther Ben Amor, Jan Knol, Johan Garssen, Jacqueline van Schaik, and Aldwin Vriesema are employees of Nutricia Advanced Medical Nutrition, Danone Research-Centre for Specialised Nutrition, Wageningen, The Netherlands. Kadija Benlhassan-Chahour and Dorothy Bray are employees of ImmunoClin Paris, France. There has been no personal financial interest of any of these people with respect to planning, executing, and reporting the study or any planned event afterwards.

\section{ACKNOWLEDGMENTS}

We acknowledge all participating patients and the COPA study team. We thank Carin Verduyn, MD, for extensive support in the design and setup of the trial and Frederike de Vries for excellent statistical analyses. This study was funded by Danone Research Centre for Specialised Nutrition, Wageningen, The Netherlands.

\section{AUTHOR CONTRIBUTIONS}

A.G., B.V.L., J.V.S., J.K., D.B., A.V., J.G., and M.C. conceived and designed the experiments; A.G., G.R., G.W., C.T., T.Q., C.T., and A.B. performed the experiments; A.G., G.R., B.V.L., K.B.A., J.V.S., J.K., D.B., A.V., J.W., J.G., and M.C. analyzed the data; K.B.A., C.T., T.Q., C.T., A.B., K.B.C., D.T., and J.W. contributed reagents/materials/analysis tools; A.G., B.V.L., and M.C. wrote the paper.

\section{REGISTRATION NUMBER AND NAME OF TRIAL REGISTER}

This study was registered in the Clinical Trial Database under ISRCTN30730587 and title Tolerance and immunological response in human immunodeficiency virus (HIV) seropositive individuals after NR100063 supplementation.

(C) 2011 Society for Mucosal Immunology 


\section{REFERENCES}

1. Brenchley, J.M. et al. CD4+ T cell depletion during all stages of HIV disease occurs predominantly in the gastrointestinal tract. J. Exp. Med. 200, 749-759 (2004).

2. Mehandru, S. et al. The gastrointestinal tract is critical to the pathogenesis of acute HIV-1 infection. J. Allergy Clin. Immunol. 116, 419-422 (2005).

3. Guadalupe, M. et al. Severe CD4+ T-cell depletion in gut lymphoid tissue during primary human immunodeficiency virus type 1 infection and substantial delay in restoration following highly active antiretroviral therapy. J. Virol. 77, 11708-11717 (2003).

4. Brenchley, J.M. et al. Microbial translocation is a cause of systemic immune activation in chronic HIV infection. Nat. Med. 12, 1365-1371 (2006).

5. Dandekar, S. Pathogenesis of HIV in the gastrointestinal tract. Curr. HIVI AIDS Rep. 4, 10-15 (2007).

6. Anthony, K.B. et al. Incomplete CD4 T cell recovery in HIV-1 infection after 12 months of highly active antiretroviral therapy is associated with ongoing increased CD4 T cell activation and turnover. J. Acquir. Immune Defic. Syndr. 33, 125-133 (2003).

7. Veazey, R.S. \& Lackner, A.A. Getting to the guts of HIV pathogenesis. J. Exp. Med. 200, 697-700 (2004).

8. Tenorio, A.R. et al. The effect of aging on T-regulatory cell frequency in HIV infection. Clin. Immunol. 130, 298-303 (2009).

9. Gori, A. et al. Early impairment of gut function and gut flora supporting a role for alteration of gastrointestinal mucosa in human immunodeficiency virus pathogenesis. J. Clin. Microbiol. 46, 757-758 (2008).

10. Saavedra, J.M., Bauman, N.A., Oung, I., Perman, J.A. \& Yolken, R.H. Feeding of Bifidobacterium bifidum and Streptococcus thermophilus to infants in hospital for prevention of diarrhoea and shedding of rotavirus. Lancet 344, 1046-1049 (1994).

11. McKenna, P. et al. The macaque gut microbiome in health, lentiviral infection, and chronic enterocolitis. PLoS Pathog. 4, e20 (2008).

12. Janoff, E.N. \& Smith, P.D. Emerging concepts in gastrointestinal aspects of HIV-1 pathogenesis and management. Gastroenterol 120, 607-621 (2001).

13. Hummelen, R., Vos, A.P., van't Land, B., van Norren, K. \& Reid, G. Altered host-microbe interaction in HIV: a target for intervention with pro- and prebiotics. Int. Rev. Immunol. 29, 485-513 (2010).

14. Lievin-Le Moal, V. \& Servin, A.L. The front line of enteric host defense against unwelcome intrusion of harmful microorganisms: mucins, antimicrobial peptides, and microbiota. Clin. Microbiol. Rev. 19, 315-337 (2006).

15. Falk, P.G., Hooper, L.V., Midtvedt, T. \& Gordon, J.I. Creating and maintaining the gastrointestinal ecosystem: what we know and need to know from gnotobiology. Microbiol. Mol. Biol. Rev. 62, 1157-1170 (1998).

16. Kelly, D., Conway, S. \& Aminov, R. Commensal gut bacteria: mechanisms of immune modulation. Trends Immunol. 26, 326-333 (2005).

17. Mazmanian, S.K., Liu, C.H., Tzianabos, A.O. \& Kasper, D.L. An immunomodulatory molecule of symbiotic bacteria directs maturation of the host immune system. Cell Mol. Biol. 122, 107-118 (2005).

18. Gibson, G.R. \& Roberfroid, M.B. Dietary modulation of the human colonic microbiota: introducing the concept of prebiotics. J. Nutr. 125, 1401-1412 (1995).

19. Vos, A.P. et al. Dietary supplementation of neutral and acidic oligosaccharides enhances Th1-dependent vaccination responses in mice. Pediatr. Allergy Immunol. 18, 304-312 (2007).

20. Moro, G. et al. Dosage-related bifidogenic effects of galacto- and fructooligosaccharides in formula-fed term infants. J. Pediatr. Gastroenterol. Nutr. 34, 291-295 (2002).

21. Arslanoglu, S. et al. Early dietary intervention with a mixture of prebiotic oligosaccharides reduces the incidence of allergic manifestations and infections during the first two years of life. J. Nutr. 138, 1091-1095 (2008).

22. Griffiths, E.A. et al. In vivo effects of bifidobacteria and lactoferrin on gut endotoxin concentration and mucosal immunity in Balb/c mice. Dig. Dis Sci. 49, 579-589 (2004).

23. Spindler-Vesel, A., Bengmark, S., Vovk, I., Cerovic, O. \& Kompan, L. Synbiotics, prebiotics, glutamine, or peptide in early enteral nutrition: a randomized study in trauma patients. J. Parenter. Enteral. Nutr. 31, 119-126 (2007).
24. Commane, D.M. et al. Effects of fermentation products of pro- and prebiotics on trans-epithelial electrical resistance in an in vitro model of the colon. Nutr. Cancer 51, 102-109 (2005).

25. Wang, Z. et al. The role of bifidobacteria in gut barrier function after thermal injury in rats. J. Trauma. 61, 650-657 (2006).

26. Cani, P.D. et al. Changes in gut microbiota control metabolic endotoxemia-induced inflammation in high-fat diet-induced obesity and diabetes in mice. Diabetes 57, 1470-1481 (2008).

27. Hunt, P.W. et al. Relationship between T cell activation and CD4+ T cell count in HIV-seropositive individuals with undetectable plasma HIV RNA levels in the absence of therapy. J. Infect. Dis. 197, 126-133 (2008).

28. Turnbaugh, P.J. et al. An obesity-associated gut microbiome with increased capacity for energy harvest. Nature 444, 1027-1031 (2006).

29. Irvine, S.L. et al. Probiotic yogurt consumption is associated with an increase of CD4 count among people living with HIV/AIDS. J. Clin. Gastroenterol. 44, e201-e205 (2010).

30. Nazli, A. et al. Exposure to HIV-1 directly impairs mucosal epithelial barrier integrity allowing microbial translocation. PLoS Pathog. 8, e1000852 (2010).

31. Cao, W., Jamieson, B.D., Hultin, L.E., Hultin, P.M. \& Detels, R. Regulatory T cell expansion and immune activation during untreated HIV type 1 infection are associated with disease progression. AIDS Res. Hum. Retroviruses 25, 183-191 (2009).

32. Azzoni, L. et al. Sustained impairment of IFN-gamma secretion in suppressed HIV-infected patients despite mature NK cell recovery: evidence for a defective reconstitution of innate immunity. J. Immunol. 168, 5764-5770 (2002).

33. Alter, G. et al. Sequential deregulation of NK cell subset distribution and function starting in acute HIV-1 infection. Blood 106, 3366-3369 (2005).

34. Eger, K.A. \& Unutmaz, D. Perturbation of natural killer cell function and receptors during HIV infection. Trends Microbiol. 12, 301-303 (2004).

35. Brunetta, E. et al. The decreased expression of Siglec-7 represents an early marker of dysfunctional natural killer-cell subsets associated with high levels of HIV-1 viremia. Blood 114, 3822-3830 (2009).

36. Vaknin, I. et al. A common pathway mediated through Toll-like receptors leads to T- and natural killer-cell immunosuppression. Blood 111, 1437-1447 (2008).

37. D'Souza, M. et al. Programmed death 1 expression on HIV-specific CD4+ $T$ cells is driven by viral replication and associated with $T$ cell dysfunction. J. Immunol. 179, 1979-1987 (2007).

38. Van Aerde, J., Alarcon, P. \& Lam, W. Tolerance and safety of energydense enteral formulae for young children. Int. Pediatr. 18, 95-99 (2003).

39. Haarman, M. \& Knol, J. Quantitative real-time PCR assays to identify and quantify fecal Bifidobacterium species in infants receiving a prebiotic infant formula. Appl. Environ. Microbiol. 71, 2318-2324 (2005).

40. Haarman, M. \& Knol, J. Quantitative real-time PCR analysis of fecal Lactobacillus species in infants receiving a prebiotic infant formula. Appl. Environ. Microbiol. 72, 2359-2365 (2006).

41. Pirnay, J.P. et al. Quantitation of Pseudomonas aeruginosa in wound biopsy samples: from bacterial culture to rapid 'real-time' polymerase chain reaction. Crit. Care 4, 255-261 (2004).

42. Nadkarni, M.A., Martin, F.E., Jacques, N.A. \& Hunter, N. Determination of bacterial load by real-time PCR using a broad-range (universal) probe and primers set. Microbiology 148, 257-266 (2002).

43. Liu, A., Schisterman, E.F., Mazumdar, M. \& Hu, J. Power and sample size calculation of comparative diagnostic accuracy studies with multiple correlated test results. Biom. J. 47, 140-150 (2005).

44. Franks, A.H. et al. Variations of bacterial populations in human feces measured by fluorescent in situ hybridization with group-specific 165 rRNA-targeted oligonucleotide probes. Appl. Environ. Microbiol. 64, 3336-3345 (1998)

45. Manz, W., Amann, R., Ludwig, W., Manz, W., Amann, R. \& Ludwig, W. Application of a suite of $16 \mathrm{~S}$ rRNA-specific oligonucleotide probes designed to investigate bacteria of the phylum cytophaga-flavobacterbacteroides in the natural environment. Microbiology 142, 1097-1106 (1996).

46. Poulsen, L.K., Licht, T.R., Rang, C., Krogfelt, K.A. \& Molin, S. Physiological state of Escherichia coli BJ4 growing in the large intestines of streptomycin-treated mice. J. Bacteriol. 177, 5840-5845 (2005).

47. Hogardt, M. et al. Specific and rapid detection by fluorescent in situ hybridization of bacteria in clinical samples obtained from cystic fibrosis patients. J. Clin. Microbiol. 38, 818-825 (2000). 
48. Harmsen, H.J. et al. Comparison of viable cell counts and fluorescence in situ hybridization using specific rRNA-based probes for the quantification of human fecal bacteria. FEMS Microbiol. Lett. 183, 125-129 (2000).

49. Zoetendal, E.G. et al. Mucosa-associated bacteria in the human gastrointestinal tract are uniformly distributed along the colon and differ from the community recovered from feces. Appl. Environ. Microbiol. 68, 3401-3407 (2002).

50. Jansen, G.J., Wildeboer-Veloo, A.C., Tonk, R.H., Franks, A.H. \& Welling, G.W. Development and validation of an automated, microscopy-based method for enumeration of groups of intestinal bacteria. J. Microbiol. Methods 37, 215-221 (1999).
51. Zoetendal, E.G., Ben-Amor, K., Akkermans, A.D., Abee, T. \& de Vos, W.M. DNA isolation protocols affect the detection limit of PCR approaches of bacteria in samples from the human gastrointestinal tract. Syst. Appl. Microbiol. 24, 405-410 (2001).

(c) This work is licensed under the Creative Commons

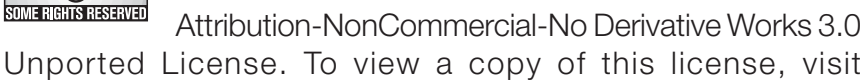
http://creativecommons.org/licenses/by-nc-nd/3.0/ 